\title{
La dialectique objets-structures comme cadre de référence pour une étude didactique du structuralisme algébrique
}

The objects-structures dialectic, a framework for a didactical study of algebraic structuralism

\section{Thomas Hausberger}

\section{OpenEdition Journals}

Édition électronique

URL : https://journals.openedition.org/educationdidactique/2750

DOI : 10.4000/educationdidactique. 2750

ISSN : 2111-4838

\section{Éditeur}

Presses universitaires de Rennes

\section{Édition imprimée}

Date de publication : 6 décembre 2017

Pagination : 131-151

ISBN : 978-2-7535-7318-5

ISSN : 1956-3485

\section{Référence électronique}

Thomas Hausberger, "La dialectique objets-structures comme cadre de référence pour une étude didactique du structuralisme algébrique », Éducation et didactique [En ligne], 11-2 | 2017, mis en ligne le 06 décembre 2019, consulté le 22 juillet 2022. URL : http://journals.openedition.org/ educationdidactique/2750 ; DOI : https://doi.org/10.4000/educationdidactique.2750 


\title{
LA DIALECTIQUE OBJETS-STRUCTURES COMME CADRE DE RÉFÉRENCE POUR UNE ÉTUDE DIDACTIQUE DU STRUCTURALISME ALGÉBRIQUE
}

\author{
Thomas Hausberger \\ Institut Montpelliérain Alexander Grothendieck, CNRS, université de Montpellier \\ E-mail : thomas.hausberger@umontpellier.fr
}

\begin{abstract}
En appui sur une étude épistémologique du structuralisme algébrique tel qu'en rendent compte les mathématiciens (Bourbaki), les historiens (Corry; Benis-Sinaceur) et les philosophes (Cavaillès; Lautman), sur l'idée de «phénoménologie didactique » des structures mathématiques introduite par Freudenthal, ainsi que sur des outils sémiotiques, nous montrons dans cet article que la dialectique objets-structures peut servir de cadre de référence pour une étude didactique du structuralisme algébrique. Ce point de vue est fertile tant pour l'élaboration d'activités mathématiques en algèbre abstraite que pour l'analyse des phénomènes cognitifs en jeu dans le processus de conceptualisation d'une structure algébrique abstraite.
\end{abstract}

Mots-clés : algèbre abstraite, structuralisme mathématique, phénoménologie didactique, dialectique objetsstructures, dialectique syntaxe-sémantique.

The objects-structures dialectic, a framework for a didactical study of algebraic structuralism

On the basis of an epistemological analysis of algebraic structuralism as accounted for by mathematicians (Bourbaki), historians (Corry; Benis-Sinaceur) and philosophers (Cavaillès; Lautman), as well as on the idea of "didactical phenomenology" of mathematical structures introduced by Freudenthal and also on semiotic tools, we argue in this paper that the objects-structures dialectic may serve as a framework of reference for a didactical study of algebraic structuralism. This point of view is fruitful both to design mathematical activities in abstract algebra and to analyse cognitive processes involved in the conceptualisation of an abstract algebraic structure.

Keywords: abstract algebra, mathematical structuralism, didactical phenomenology, dialectic of objects and structures, dialectic of syntax and semantics. 
Thomas Hausberger

\section{INTRODUCTION}

Ce travail s'inscrit dans la problématique de l'enseignement et de l'apprentissage de l'algèbre abstraite à l'Université. Nous désignons sous ce vocable la discipline dédiée à l'étude des structures algébriques, c'est-à-dire à l'investigation des conséquences logiques de systèmes axiomatiques spécifiques particulièrement fertiles et des relations entre ces systèmes. Les difficultés sont reconnues par de nombreux auteurs (Leron \& Dubinsky, 1995; Nardi, 2000 ; Lajoie \& Mura, 2004; Hausberger, 2013; Durand-Guerrier, Hausberger $\&$ Spitalas, 2015) et reflètent un problème de "transition » (Gueudet, 2008), qui a lieu cette fois, par comparaison avec la transition lycée-université, à l'intérieur d'une même institution, à la transition entre la licence et le master de mathématiques.

Les premiers éléments de notre étude épistémologique (Hausberger, 2013) ont permis de relier ce phénomène à la transition épistémologique suscitée par « the systematization of the axiomatic method, after Hilbert, and the transition, after Noether, from thinking about operations on elements to thinking in terms of selected subsets and homomorphisms $»$ (Hausberger, 2013, p. 2354). En effet, ainsi que le souligne Corry (2016):

\footnotetext{
"Van der Waerden's new image of the discipline inverted the conceptual hierarchy of classical algebra. Groups, fields, rings, and other related concepts became the main focus, based on the implicit realization that all of these concepts were, in fact, instances of a more general, underlying idea: the idea of an algebraic structure" (paragr. 1)
}

En d'autres termes, le saut épistémologique conduit à une disparition des objets concrets au profit de structures abstraites surplombantes. De plus, l'étude de la transposition didactique de la notion de structure (Hausberger, 2013) montre qu'il s'agit d'un métaconcept qui n'est défini mathématiquement dans aucun cours ni manuel de niveau licence ou début de master (et ne peut l'être). «As a consequence, students are supposed to learn by themselves and by the examples what is meant by a structure whereas sentences like " $a$ homomorphism is a structure-preserving function" is supposed to help them make sense of a homomorphism " (Hausberger, 2013, p. 2352). Ces éléments nous ont conduits à regarder plus finement les rapports entre objets et structures dans la pensée structuraliste, avec pour but de développer une « didactique du structuralisme algébrique », de même qu'il existe une didactique de l'algèbre élémentaire. Chevallard (1989) a souligné la dialectique entre l'arithmétique et le calcul algébrique en l'interprétant en terme d'articulation syntaxe-sémantique. Existerait-il de la même manière en algèbre abstraite un rapport dialectique entre objets et structures, qui s'apparenterait également à une dialectique entre syntaxe et sémantique? Ce point de vue serait-il fécond pour les analyses didactiques, comme c'est le cas en algèbre classique (Kouki, 2008)?

Dans notre contexte, les systèmes axiomatiques abstraits constituent le point de vue syntaxique par rapport aux objets concrets qui en fournissent différentes sémantiques. Une dialectique concretabstrait est donc également en jeu. Par ailleurs, le constat épistémologique précédent (la disparition des objets au profit des structures) laisse à penser que l'enseignement de l'algèbre abstraite aura tendance à manifester un déficit sémantique suite à une prédominance des aspects syntaxiques. Quel est le rôle et l'importance des aspects sémantiques en algèbre abstraite? Notre didactique du structuralisme algébrique doit permettre de rendre compte du processus de conceptualisation d'une structure abstraite, en relation avec les processus d'abstraction qui sont en jeu. Nous faisons l'hypothèse que l'articulation entre syntaxe et sémantique, à travers la dialectique entre objets et structures, est fondamentale à cet égard.

Afin de répondre à ces questions et d'étayer nos hypothèses, nous avons poursuivi nos analyses épistémologiques, en appui sur le travail des historiens et des philosophes. Nous introduirons tout d'abord des éléments d'épistémologie historique sur le structuralisme mathématique puis nous présenterons les grandes dialectiques mises en évidence par les philosophes Lautman et Cavaillès afin de rendre compte de la genèse des idées mathématiques structuralistes. Ceci nous permet de placer au cœur de la pensée structuraliste la dialectique entre objets et structures, prise en deux grands mouvements d'abstraction : l'idéalisation et la thématisation. Nous nous employons ensuite à constituer cette dialectique épistémologique en une dialectique didactique. Pour cela, nous nous tournons vers l'idée, due à Freudenthal, de « phénoménologie des structures mathématiques » et étendons à l'algèbre abstraite l'usage qui en est fait dans le cas de la didactique de l'algèbre élémentaire. 
Cela nous conduit à introduire de nouveaux outils sémiotiques qui permettent d'éclairer notamment le processus d'idéalisation dans ses aspects syntaxiques et sémantiques.

Nous présentons en définitive, dans cet article, un cadre de référence pour une étude didactique du structuralisme algébrique, construit en croisant des approches historique, épistémologique, philosophique, didactique et cognitive. Il est destiné à l'élaboration d'ingénieries en algèbre abstraite ainsi qu'à l'analyse des phénomènes cognitifs en jeu dans le processus de conceptualisation d'une structure algébrique abstraite. Nous évoquerons dans la conclusion, en guise de prolongements, un exemple de tels travaux.

\section{LE STRUCTURALISME ALGÉBRIQUE}

\section{L'héritage de Hilbert et Noether}

L'algèbre structuraliste est le fruit des mathématiques abstraites qui, dans le premier tiers du $\mathrm{XX}^{\mathrm{e}}$ siècle, se développèrent dans l'école allemande de David Hilbert et Emmy Noether. Nous rendons compte dans ce paragraphe de cet héritage, en nous appuyant sur les travaux de Corry (1996) et BenisSinaceur (2010, mars). Ces éléments sont repris de Hausberger (2012).

L'algèbre désigne traditionnellement un ensemble d'idées et de techniques mathématiques associées à la manipulation formelle de symboles abstraits, en lien avec la résolution des équations. L'algèbre moderne est née de ce terreau mais se caractérise par un changement de perspective (de paradigme?) qui se traduit par une restructuration-refondation mathématique de son champ et une diffusion de ses méthodes à d'autres branches des mathématiques. Ces dernières se trouvent ainsi unifiées et participent de la filiation de l'algèbre moderne : c'est le cas de l'arithmétique et de la géométrie notamment, plus tardivement de la topologie. Ce processus d'abstraction présente historiquement différentes étapes identifiables.

Les groupes de substitutions apparaissent tout d'abord comme des outils pour décider de la résolubilité par radicaux des équations et pour mettre en œuvre cette résolution par extractions successives de racines (travaux de Galois). Le premier emploi du mot groupe est alors proche de son sens commun de regroupement d'éléments : aucune définition n'est donnée. Les groupes s'autonomisent progressivement en tant qu'objets d'étude, indépendamment du contexte de la résolution des équations. Mais un grand nombre de notions de théorie des groupes (groupe distingué, groupe simple, suite de décomposition) sont directement reliées à cette application. Pour Jordan, les groupes de substitutions et leurs quotients ne font pas partie de la même théorie (c'est Hölder qui mettra au point les groupes quotients et montrera que les groupes distingués sont la bonne notion pour faire des quotients, aboutissant au résultat connu actuellement sous le nom de théorème de Jordan-Hölder).

L'origine des groupes abéliens se situe en arithmétique avec les travaux de Gauss sur la composition des formes, présentés dans les Disquisitiones arithmeticae. Une chaîne logique de développements conduit des travaux de Gauss à l'axiomatisation (implicite) par Kronecker des groupes abéliens finis et la démonstration de leur théorème de structure. Pour autant, Kronecker n'utilise pas le terme de groupe, car il ne fait pas le lien avec les groupes de substitutions (ceci sera réalisé peu après par son étudiant Eugen Netto).

L'évolution historique du concept de groupe s'est faite en relation avec ses domaines d'application. La communauté mathématique ne s'est emparée du concept de groupe abstrait, issu des travaux de Cayley autour de la notion de générateur, qu'après l'apparition des groupes dans d'autres contextes: outre en arithmétique, celle des groupes de transformation et la reconnaissance du rôle des groupes dans l'unification des géométries (programme d'Erlangen de Klein).

Un facteur favorable est à trouver dans les papiers de Boole sur l'algèbre formelle qui contribue à installer un point de vue abstrait sur l'algèbre. L'idée que deux groupes isomorphes sont essentiellement les mêmes a pu alors émerger et van Dyck met au point la présentation par générateurs et relations (en quotientant le groupe libre) dans le but d'unifier les trois sources historiques des groupes autour d'une même présentation. La reconnaissance de son rôle central en mathématiques a permis le développement de la structure de groupe, première structure algébrique abstraite, ceci avant le début du $\mathrm{XX}^{\mathrm{e}}$ siècle.

La transition vers la structure abstraite de groupe a lieu également en relation avec la généralisation de la méthode axiomatique en mathématiques prônée par Hilbert : d'une part, la présentation axiomatique des 
théories s'impose de plus en plus dans la communauté mathématique eu égard à la question des fondements, la théorie des ensembles fournissant la base de l'édifice; d'autre part, l'axiomatique fait office d'outil didactique pour produire une présentation plus commode et intelligible d'un contenu structuré, à même d'isoler de l'ensemble des propriétés des objets celles qui découlent du fait que ces derniers vérifient les axiomes de structure préalablement dégagés.

L'unification produite a lieu à différents niveaux. $\mathrm{Au}$ premier niveau, une même théorie (la théorie des groupes) s'applique à des objets de natures différentes. À un niveau supérieur, la présentation axiomatique des structures algébriques permet un traitement unifié mettant en avant les ponts entre ces structures. C'est le point de vue novateur du traité de van der Waerden (1930-1931), héritier du style d'Emmy Noether. Ce traitement unifié des structures marque l'avènement de la pensée structuraliste et transforme l'algèbre en une discipline dédiée à l'investigation de structures, à propos desquelles on se pose le même type de questions que l'on cherche à résoudre avec le même type d'outils. Les origines de la pensée structuraliste remontent à Galois, dont la mise en forme des idées a été l'un des moteurs, comme le montre le traité de Weber (1898). Cependant, sa mise en forme jusqu'à la présentation actuelle est le fruit d'un long processus de conceptualisation. L'idée des structures algébriques comme principe unificateur est absent des écrits de Hilbert et coïncide bien avec l'apport de Noether.

L'expression de "mathématique conceptuelle » est utilisée par un certain nombre de mathématiciens allemands des $\mathrm{XIX}^{\mathrm{e}}$ et $\mathrm{XX}^{\mathrm{e}}$ siècles (Riemann, Hasse, Dedekind, Hilbert, Noether) pour caractériser leur méthode de travail, focalisée sur la formation des concepts, et que certains historiens font remonter à Gauss. Plus que par le type d'objet étudié, la pensée structuraliste se caractérise donc par une méthodologie et un style spécifiques, qui font école à Göttingen autour de Noether. Cette école change la manière de prouver en privilégiant les preuves générales limitant les calculs et mettant en avant les concepts. Définir des concepts a pour objectif de reconstruire un domaine sur une nouvelle base, sur la base de concepts plus fondamentaux, plus généraux et plus « simples». Comme l'indique Helmut Hasse (1930, p. 26-27), il s'agit de « s'appliquer à réduire un domaine mathématique à ses concepts fondamentaux les plus généraux, donc les plus simples, puis à construire et à reconstruire à l'aide de ces seuls concepts $»$. Il est donc question d'une refondation mathématique (et non philosophique). Cette reconstruction apporte une vision nouvelle de la matière mathématique et ouvre la voie à des constructions inédites, à de nouveaux objets. Ces concepts permettent de constituer la théorie d'un domaine, c'est-à-dire de distinguer les propriétés transférables ou généralisables à d'autres domaines de celles qui ne le sont pas. Sous la plume de Noether, concepts et structures se confondent souvent. Les structures algébriques sont donc le fruit d'un projet mené par des mathématiciens portant une conception particulière des mathématiques, adoptée après une réflexion sur leur propre activité. La pensée structuraliste va de pair avec un questionnement métamathématique, dont on peut trouver des traces dans les correspondances entre mathématiciens ainsi que dans les préfaces des traités et manuels ${ }^{1}$.

\section{Carnap et la « logique des relations »}

Nous présentons dans cette section, en suivant Patras (2008), un point de vue logique sur la notion de relation telle qu'elle apparaît sous la plume de Carnap (1928). Ce point de vue est éclairant dans les distinctions qu'il permet de saisir relativement au processus d'abstraction en jeu dans l'algèbre abstraite.

Carnap est assez proche dans ses écrits de la conception hilbertienne de l'axiomatique (une axiomatique formelle faisant abstraction de la nature particulière des éléments afin de mettre en avant les relations entre objets). Il revendique également sa dette à l'égard des logiciens Russell et Whitehead, ce qui l'amène à introduire la distinction entre " description de propriété ", " description de relation » et « description de structure » (Carnap, 1928, cité dans Patras, 2008) :

«Pour développer le concept de structure, fondamental pour la théorie des constructions, nous faisons une distinction entre deux types de descriptions d'objets d'un domaine quelconque. Une description de propriété indique les propriétés que possèdent les objets individuels d'un domaine donné, tandis qu'une description de relation indique les relations qui ont lieu entre ces objets, sans affirmer quoi que ce soit sur ces objets en tant qu'individus. Ainsi, une description de 
propriété fait des assertions individuelles ou, en un sens, absolues, tandis qu'une description de relation fait des assertions relatives [...]. Les descriptions de relations forment le point de départ de tout le système constructionnel et constituent donc la base de la science unifiée [...]. Parmi les descriptions de relations, il en est un certain type, que nous appellerons description de structure. À l'encontre des descriptions de relations, celles-ci ne laissent pas seulement les propriétés des éléments du domaine non mentionnées : en fait, elles ne spécifient pas même les relations elles-mêmes qui existent entre ces éléments. Dans une description de structure, seule la structure de la relation est indiquée, c'est-à-dire, la totalité de ses propriétés formelles. Par propriété formelle d'une relation, j'entends une propriété qui peut être formulée sans référence à la signification de la relation et aux types d'objets qu'elle concerne. » (p. 46-47.)

L'axiomatique d'une structure algébrique, que nous considérons comme un point de vue syntaxique sur la structure, présente ainsi différents niveaux d'abstraction : s'il s'agit bien de décrire de façon abstraite des propriétés d'objets d'un domaine concret, en mettant en avant les relations, la description de ces relations fait apparaître un second niveau d'abstraction : il faut décrire les propriétés formelles de ces relations indépendamment de leur sémantique (par exemple les trois propriétés de réflexivité, symétrie et transitivité définissant une relation d'équivalence). C'est seulement lorsque ce second niveau est atteint que l'on obtient une description de structure. En définitive, une première étape fait abstraction de la nature des objets et une seconde étape de la sémantique des relations.

Il est important de bien saisir la difficulté conceptuelle sous-jacente à cette idée de "structure des relations » : la présentation axiomatique formelle (un système axiomatique définissant une structure met en avant la logique des relations, c'est-à-dire leurs propriétés formelles) est peu propice à développer des images mentales alors même que l'étymologie du mot relation contient de façon naturelle l'idée de relier des éléments. La pluralité des axiomes est le résultat d'un processus d'analyse alors que le point de vue relationnel relève sémantiquement d'un point de vue synthétique. Carnap, qui était déjà sensible à ces difficultés, a recours à une représentation mentale particulière afin d'expliquer cette idée de "structure de relations » (cité dans Patras, 2008) :
« Pour comprendre ce que l'on entend par la structure d'une relation, pensons au diagramme de flèches suivant : représentons tous les membres de la relation par des points. De chaque point, une flèche va vers les autres points qui lui sont en relation. Une flèche double désigne les paires pour lesquelles la relation vaut dans les deux directions. Une flèche qui retourne à son origine désigne un membre en relation avec lui-même. Si deux relations ont le même diagramme de flèches, on les dit structurellement équivalentes, ou isomorphes. Le diagramme de flèches est la représentation symbolique de la structure. » (p. 49.)

La représentation symbolique de la structure d'une relation en termes de diagrammes de flèches est ce qu'on appelle aujourd'hui une représentation par un graphe mathématique. La théorie des graphes s'est développée à un rythme très rapide dans le dernier tiers du XXe siècle en lien avec l'informatique, où la théorie des graphes joue un rôle important. Un graphe apparaît pour décrire une situation mathématique dès que celle-ci implique un schéma de dépendances (entre éléments) suffisamment complexe pour ne pas être réductible à une notion classique comme celle de fonction. La théorie des graphes fournit ainsi un symbolisme particulier, ancré dans l'image mentale de «lien entre éléments ». Ce symbolisme offre un point de vue abstrait synthétique (auquel la force de la représentation de nature géométrique n'est pas étrangère) sur la logique des relations, comparativement au point de vue axiomatique logique qui est un point de vue abstrait analytique. Il permet de développer une combinatoire des relations en appui sur des processus cognitifs engageant les sens, donc sur la phénoménologie.

Carnap ne s'arrête pas là et introduit une notion d'isomorphisme entre deux systèmes d'objets munis d'une relation, ce qui lui permet de définir une notion de structure :

«Une extension de relation $\mathrm{R}$ est appelée un corrélateur entre deux extensions de relations $\mathrm{P}$ et $\mathrm{Q}$ si elle établit une correspondance biunivoque entre les éléments de $\mathrm{P}$ et ceux de $\mathrm{Q}$, de telle sorte qu'à chaque paire de $\mathrm{P}$ correspond une paire de $\mathrm{Q}$ et vice-versa ${ }^{2}$. Si un tel corrélateur existe pour deux extensions de relation $\mathrm{P}$ et $\mathrm{Q}$, alors $\mathrm{P}$ et $\mathrm{Q}$ sont dites isomorphes ou de même structure [...]. Nous pouvons maintenant donner la définition exacte de la structure d'une extension de 
relation P : c'est la classe des extensions de relation qui sont isomorphes à P. » (p. 49.)

Carnap utilise ici la notion d'extension de concept, au sens de Frege, et le « principe d'abstraction », introduit par Russell, que nous détaillerons plus loin ${ }^{3}$. L'extension d'une relation désigne ainsi la totalité des objets qui tombent sous le concept défini par la relation, ou encore son domaine. Le corrélateur établissant une relation d'équivalence, Carnap définit la structure comme une classe (d'isomorphisme).

\section{Bourbaki et le concept de structure mathématique}

Dans son travail d'historien, Corry (1996) distingue le « contenu du savoir» (body of knowledge) et l'« image du savoir» (image of knowledge). Le concept de "structure mathématique » appartient à l'image du savoir. À propos de l'ouvrage Moderne Algebra de van der Waerden (1930-1931), il écrit :

"This textbook put forward a new image of the discipline that implied in itself a striking innovation: the structural image of algebra. In the forthcoming account, it is this specific, historically conditioned image of mathematical knowledge that will be considered as implicitly defining the idea of a mathematical structure" (p.8).

Le collectif de mathématiciens Bourbaki fut l'un des grands propagateurs de cette "image structurale ", comme en témoigne le manifeste "L'architecture des mathématiques » (Bourbaki, 1948/1998). Son traité Les Éléments de mathématique (publié à partir de 1939) est marqué par une volonté d'extension à l'ensemble des mathématiques de la vision structurale unificatrice de Noether. Bourbaki y définit mathématiquement la notion de structure, mais sa définition ne sert que de cadre général et n'est pas mathématiquement " fonctionnelle » (Corry, 1996, p. 324), à la différence de la théorie des catégories, introduite par Mac Lane et Eilenberg (Mac Lane, 1996). Cette dernière constitue une véritable métathéorie mathématique des structures, mais il s'agit d'un point de vue très surplombant, qui est hors d'atteinte, par exemple, des étudiants lorsqu'ils apprennent la théorie des structures algébriques. En définitive, pour les mathématiciens du milieu $\mathrm{du} \mathrm{XX} \mathrm{X}^{\mathrm{e}}$ siècle tout comme pour les étudiants (mis à part les étudiants de deuxième année de master qui se destinent à la recherche dans ce domaine précis des mathématiques), le concept de structure, faute de fondation mathématique, appartient essentiellement à « l'image du savoir ».

Bourbaki donne dans son manifeste (1948/1998) une définition informelle de la notion de structure :

«On peut maintenant faire comprendre ce qu'il faut entendre, d'une façon générale, par une structure mathématique. Le trait commun des diverses notions désignées sous ce nom générique, est qu'elles s'appliquent à des ensembles d'éléments dont la nature n'est pas spécifiée; pour définir une structure, on se donne une ou plusieurs relations, où interviennent ces éléments [...] ; on postule ensuite que la ou les relations données satisfont à certaines conditions et qui sont les axiomes de la structure considérée. Faire la théorie axiomatique d'une structure donnée, c'est déduire les conséquences logiques des axiomes en s'interdisant toute autre hypothèse sur les éléments considérés (en particulier toute hypothèse sur leur "nature" propre). » (p. 40-41.)

Il est intéressant de noter qu'il s'agit davantage de la description d'une méthode qu'une véritable définition. Si le structuralisme est fondé sur la construction de concepts (comme ceux de groupe, d'anneau, de corps, qui sont des exemples de structures), le métaconcept de structure est donc à vocation davantage méthodologique que définitionnel. Il prend sens dans l'action.

La citation précédente de Bourbaki fait également écho à la « logique des relations » de Carnap : il s'agit du même procédé d'abstraction, lequel conduit à l'écriture d'axiomatiques formelles. Cependant Bourbaki insiste sur le rôle des intuitions et il ne s'agit donc pas de voir dans la méthode structuraliste une réduction des mathématiques à l'application mécanique de règles logiques :

«Or, chaque structure apporte avec elle son langage propre, tout chargé des résonances intuitives particulières, issues des théories d'où l'a dégagée l'analyse axiomatique que nous avons décrite plus haut; et pour le chercheur qui brusquement découvre cette structure dans les phénomènes qu'il étudie, c'est comme une modulation subite orientant d'un seul coup dans une direction inattendue le courant intuitif de sa pensée, et éclairant d'un jour nouveau le paysage mathématique 
où il se meut. [...] C'est dire que, moins que jamais, la mathématique est réduite à un jeu purement mécanique de formules isolées; plus que jamais, l'intuition règne en maîtresse dans la genèse des découvertes; mais elle dispose désormais des puissants leviers que lui fournit la théorie des grands types de structures, et elle domine d'un seul coup d'œil d'immenses domaines unifiés par l'axiomatique, où jadis semblait régner le plus informe chaos. » (p. 42-43.)

Ce que Bourbaki appelle « résonances intuitives particulières » fait directement écho à la « phénoménalité des structures mathématiques » dont il sera question plus loin lorsque nous présenterons la thèse de Freudenthal. Mais avant de développer les aspects cognitifs, analysons tout d'abord la pensée structuraliste à la lumière des grandes dialectiques mises en évidence par les philosophes. Ceci nous conduira à poser la dialectique objets-structures en tant que fondement épistémologique du structuralisme algébrique, puis de la constituer en tant que dialectique didactique.

\section{I A L E C T Q UES MATHÉMATIQUES, DIALECTIQUES DIDACTIQUES}

\section{Dialectiques mathématiques selon Lautman}

D'après Benis-Sinaceur (2010), Lautman introduit la notion de "dialectiques mathématiques » dans son «Essai sur les notions de structure et d'existence en mathématiques » (Lautman, 2006, cité dans BenisSinaceur, 2010):

« Des résultats partiels, des rapprochements arrêtés à mi-chemin, des essais qui ressemblent encore à des tâtonnements, s'organisent sous l'unité d'un même thème et laissent apercevoir dans leur mouvement une liaison qui se dessine entre certaines idées abstraites, que nous proposons d'appeler dialectiques. » (p. 40.)

Le caractère dialectique de cette « liaison » s'explique par la présence de couples d'opposés, donc de dualités dans le fonctionnement interne des mathématiques (Lautman, 2006, cité dans Benis-Sinaceur, 2010):

« La dialectique mathématique est essentiellement constituée par la dualité entre entités complémentaires ou même convertibles l'une en l'autre par des procédures inversibles. La dialectique est le passage d'un aspect à l'autre, d'un point de vue à un autre, et la coexistence de ces différents aspects dans le même être, ou plutôt dans le même devenir. » (p. 41.)

Le projet de Lautman est de « dégager une philosophie des genèses mathématiques " (Lautmann 2006, cité dans Benis-Sinaceur, 2010, p. 44). Il considère d'une part une genèse mathématique interne, d'autre part une genèse métaphysique des notions mathématiques, dont nous ne parlerons pas ici. Benis-Sinaceur (2010) rend compte de la genèse interne en ces termes ${ }^{4}$ :

« Elle est une "procession des êtres mathématiques les uns à partir des autres". Elle se fait dans l'indissoluble association du concret et de l'abstrait, de la structure et de l'existence, du domaine global et des êtres distingués, en un mot de la matière et de la forme. Lanalyse des "mixtes" a pour conséquence "l'engagement du concret dans la genèse de l'abstrait" par l'instauration d'une relation "d'imitation [...] entre la structure de cet abstrait et celle du concret de base". Réciproquement il y a aussi engagement de l'abstrait dans la genèse du concret, de la structure à la découverte de modèles inattendus ou d'êtres nouveaux. Par exemple, les représentations linéaires d'un groupe abstrait constituent un passage de la structure à l'existence. » (p. 44.)

Les grandes dialectiques lautmaniennes sont les suivantes : concret / abstrait, structure / existence, local / global, ce que le philosophe subsume sous la dialectique matière / forme. Lautman rejoint en cela les analyses de Cavaillès (1994) sur les rapports entre forme et contenu lorsqu'il écrit (Lautman, 2006, cité dans Benis-Sinaceur, 2010, p. 34) : «l'achèvement $[\ldots]$ s'affirme dans son pouvoir créateur : [...] l'essence d'une forme se réalisant dans une matière qu'elle créerait, l'essence d'une matière faisant naître les formes que sa nature dessine. »

La notion d'opération est importante dans ce rapport de la forme à la matière (Lautman, 2006, cité dans Benis-Sinaceur, 2010, p. 33) : « La solidarité entre [un] domaine et les opérations possibles sur ce domaine met au premier plan de la recherche mathématique la liaison entre opérations abstraites et domaine concret $»$. Pour Granger (1994), il y a une corrélation entre opérations et objets, corrélation qui provient du fonctionnement même de la pensée 
symbolique, et qui rend compte du rapport formecontenu mis en avant par Cavaillès. Cela le conduit à introduire le triptyque formes-opérations-objets.

À la suite de Lautman, nous plaçons au cour de notre modèle épistémologique de référence pour l'algèbre abstraite une dialectique que nous appellerons la dialectique objets-structures. Comme nous l'avons $\mathrm{vu}$, elle est fortement liée à la dialectique concretabstrait, cas particulier d'une dialectique matièreforme, et elle est à examiner en relation avec la notion d'opération (en fait de relation, au sens de la logique des relations précédemment évoquée).

\section{La dialectique objets-structures : idéalisation et thématisation selon Cavaillès}

Cavaillès appelle " dialectique des concepts » cette dialectique matière-forme qu'il voit à l'œuvre dans l'« expérience mathématique » des années trente (tout comme Lautman, Cavaillès a séjourné à Göttingen), marquée par une irrésistible montée vers l'abstraction (Benis-Sinaceur, 1987) :

«Les concepts, schémas résultant de processus d'abstraction de différents ordres et s'exerçant selon diverses directions sur des contenus plus ou moins complexes, sont des jalons dans le mouvement indéfini qui se ressource en eux pour un nouveau départ, vers de nouveaux enchaînements. » (p. 28-29.)

La dialectique des concepts est la dialectique par laquelle « l'idée » et « l'idée de l'idée » manifestent leur «puissance génératrice ». Pour préciser cette pensée, il s'agit de rendre compte des deux grands mouvements d'abstraction en jeu, l'un vertical, l'autre horizontal, que Cavaillès appelle «idéalisation » et " thématisation », dans la ligne de Husserl, et qui deviendront pour lui des " propriétés constitutives de l'essence de la pensée » (Benis-Sinaceur, 1987):

«L'idéalisation" "consiste simplement à exiger qu'une opération, qui se trouvait d'une manière accidentelle limitée à certaines circonstances extrinsèques à l'accomplissement même de cette opération, soit libérée de cette limitation extrinsèque, et ceci par la position d'un système d'objets qui ne coïncide plus avec les objets de l'intuition. C'est par exemple ainsi que sont faites les différentes généralisations de la notion de nombre." » (p. 24.)

\section{Benis-Sinaceur (1987) précise encore :}

«Lidéalisation, c’est le passage, nécessairement progressif, étalé sur une certaine "longueur d'enchaînement", de l'acte - additionner des nombres entiers, par exemple - au sens - additionner sans spécification d'individus - de la "liaison-acte" à la "liaison-type"». (p. 24.)

En termes mathématiques, il s'agit du processus d'axiomatisation-formalisation, qui consiste à exprimer les propriétés formelles des relations dans le langage symbolique de l'algèbre abstraite.

Cavaillès appelle ensuite "thématisation »le fait que « les gestes accomplis sur un modèle ou un champ d'individus peuvent, à leur tour, être considérés comme des individus sur lesquels le mathématicien travaille en les considérant comme un nouveau champ » (cité dans Benis-Sinaceur, 1987, p. 24). Prenant la structure (les relations entre objets) comme objet, le mathématicien travaille donc, dans cette phase de thématisation, à l'élaboration d'une théorie d'objets de niveau supérieur. Citant encore Cavaillès, Benis-Sinaceur, précise :

« La "thématisation", elle, "prend pour départ l'enchaînement saisi cette fois dans son vol, trajectoire qui se mue en sens. La pensée ne va plus vers le terme créé mais part de la façon de créer pour en donner le principe par une abstraction de même nature que l'autre, mais dirigée transversalement" (...). Ce deuxième processus est conditionné par le premier. Car, dans le passage de l'acte au sens apparaît une dualité : entre le sens d'une opération en tant qu'elle est opérée et son sens en tant qu'opérande. » (p. 24.)

À travers son analyse phénoménologique des processus en jeu dans l'algèbre abstraite, Cavaillès vise des " propriétés constitutives de l'essence de la pensée », ce qui diffère de notre projet restreint à l'analyse du savoir mathématique en lien avec les apprentissages. Cependant, la réflexion du philosophe attire notre attention sur le fait que cette thématisation est indissociable d'une réflexion « méta » sur le processus d'idéalisation lui-même, ce qui constitue une seconde forme d'abstraction que l'on peut rapprocher du processus d'abstraction réfléchissante de Piaget (Piaget \& Beth, 1961) : la pensée, dans la thématisation, vise le processus d'abstraction-axiomatisation lui-même pour en abstraire 
Figure 1.

Les différents niveaux de formes-objets impliqués dans la pensée structuraliste

Espace de formes

Théorie d'objets

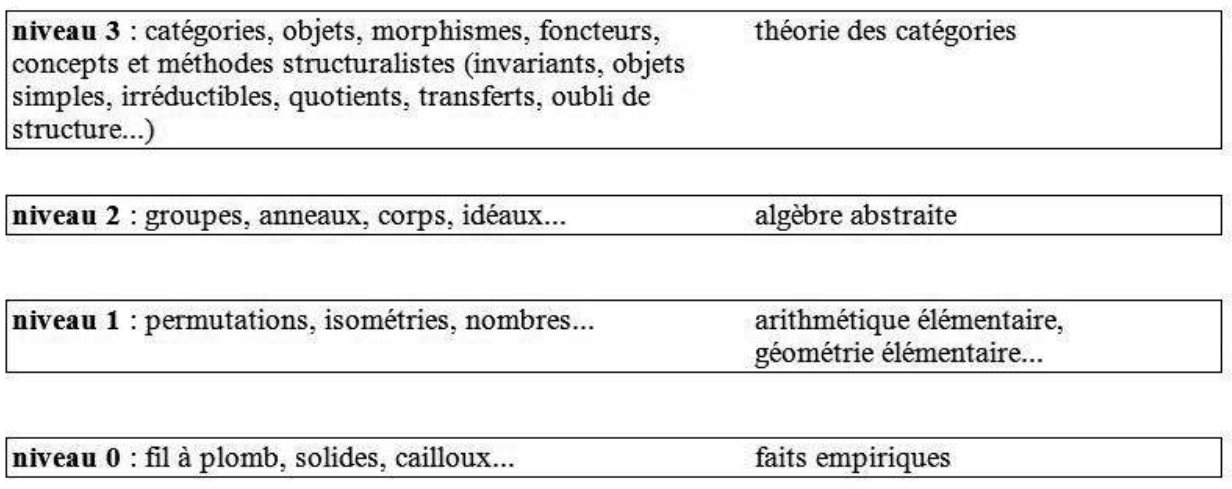

Figure 2.

Les différentes dynamiques de la pensée structuraliste

a) Dynamique verticale:

\begin{tabular}{ll|}
\hline niveau $i+1$ (structure) \\
niveau $i$ (objets) \\
\hline
\end{tabular}

b) Dynamique horizontale:

\begin{tabular}{|lll|}
\hline & thématisation & théorie d'objets \\
$\longrightarrow$
\end{tabular}

c) Dynamique globale:

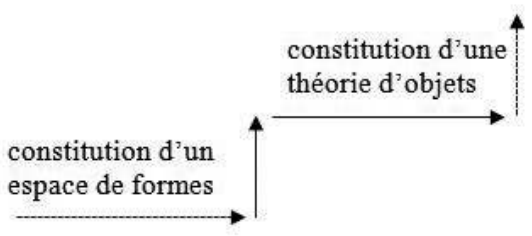

la méthode. Il s'agit d'un niveau « méta », destiné à comprendre le sens de la démarche axiomatique structuraliste, en tant qu'elle est opératoire sur des objets. C'est en effet une réflexion sur leur propre méthode qui a conduit les algébristes allemands à la réécriture de l'algèbre sous la forme que l'on connaît de nos jours.

Ainsi que le souligne Benis-Sinaceur (1987, p. 29) à la suite de Cavaillès, les deux mouvements d'idéalisation et de thématisation ne cessent pas de se succéder : « Le processus de "thématisation" superpose indéfiniment les formes-principes aux formes-opérations, les structures-concepts aux procédures idéalisées ». Il en résulte une organisation de la matière mathématique en différents " niveaux » de formesobjets, dont nous proposons la schématisation suivante (Fig. 1) :

Cette schématisation (Fig. 1) nous permet également de représenter les deux mouvements d'abstraction de Cavaillès, lesquels s'exercent transversalement : la pensée structuraliste se meut selon une dynamique à deux composantes, l'une verticale 
qui conduit à l'idéalisation d'un espace de formes, l'autre horizontale qui consiste en la thématisation de cet espace en une théorie d'objets (Fig. 2, a et b).

Le dernier schéma (Fig. 2, c), qui s'apparente à celui d'un « escalier vers l'abstraction », illustre ainsi un processus graduel qui est philosophiquement fondé et dont les enjeux cognitifs sont grandement liés à la prise en compte de ce double mouvement impliquant plusieurs niveaux de formes-objets-théories. Les descriptions précédentes ont également mis en avant des enjeux dialectiques : si ce schéma simplifié des différentes dynamiques met en avant une abstraction croissante, les mécanismes de pensée en jeu sont fondés sur une dialectique concretabstrait. Ainsi le rapport entre objets et structures est-il dialectique, et la thématisation est-elle à voir comme une méthode appliquée sur une pluralité d'espaces de formes, dans un traitement unifié des structures et en lien avec le processus d'idéalisation.

\section{Dialectiques didactiques}

Les didacticiens font également usage du terme « dialectique ». Ainsi la « dialectique outil-objet» est-elle définie en tant que " processus cyclique organisant les rôles respectifs de l'enseignant et des élèves, au cours duquel les concepts mathématiques jouent alternativement le rôle d'outil pour résoudre un problème et d'objet prenant place dans la construction d'un savoir organisé » (Douady, 1986, p. 5). La théorie anthropologique du didactique dénombre également un certain nombre de dialectiques dans la mise en œuvre de la "pédagogie de l'enquête * (Chevallard, 2001) : les dialectiques « du sujet et du hors-sujet ", « du parachutiste et du truffier », « des boîtes noires et des boîtes claires », " de la conjecture et de la preuve », « de l'excription et de l'inscription », « de la diffusion et de la réception ». Citons également la dialectique "syntaxe-sémantique " mise en évidence par les logiciens et dont la pertinence pour les analyses didactiques a été soulignée par les travaux de Durand-Guerrier (2003). Le point commun entre ces acceptions est à trouver dans un processus cyclique qui s'effectue entre deux pôles complémentaires. Chevallard (2001, p. 185) parle de « tensions bipolaires que l'on nommera, pour cela, des dialectiques $»$.

Ces diverses dialectiques ne se situent pas toutes sur le même plan : elles peuvent être suscitées par un mouvement dialectique inhérent au déploiement de la pensée mathématique, donc du savoir lui-même, dans son élaboration (dialectique interne), ou bien au niveau du fonctionnement cognitif d'un individu en réaction au milieu (dialectique épistémique), ou bien au niveau de l'action didactique de l'enseignant en vue de produire un certain effet sur l'apprentissage (dialectique didactique). Bien que la dialectique outilobjet ait été utilisée par certains didacticiens afin de commenter la genèse historique de certains concepts, dans un rapprochement entre genèses historique et expérimentale, il s'agit essentiellement d'une dialectique didactique. Les dialectiques de Chevallard sont essentiellement épistémiques, mais elles vont requérir une intervention didactique car « dans la culture didactique scolaire actuelle, elles apparaissent encore largement bloquées » (Chevallard, 2001, p. 186), ce qui en fera également des dialectiques didactiques. Les différents types de dialectiques se retrouvent ainsi reliés dans le phénomène complexe de transmission des connaissances, c'est-à-dire le phénomène didactique.

Les dialectiques objets-structures et concretabstrait qui nous concernent sont, nous l'avons souligné, des dialectiques internes au déploiement de la pensée structuraliste. Ce sont également des dialectiques épistémiques: l'évocation des objets mentaux (fruits cognitifs de ces dialectiques) et les rapports à la phénoménologie en témoignent. Notre principale préoccupation est maintenant de les constituer en de véritables dialectiques didactiques, c'est-à-dire d'étudier les modalités et les conditions de leur mise en œuvre en classe dans des situations d'apprentissage à même de favoriser le processus de conceptualisation d'une structure algébrique abstraite. Cela nous amènera à considérer également la dialectique syntaxe-sémantique dont nous montrerons bientôt l'importance dans le cadre de l'enseignement et l'apprentissage des structures algébriques.

En termes cognitifs, la description de l'idéalisation donnée par Benis-Sinaceur à la suite de Cavaillès fait écho à un processus d'encapsulation d'un acteprocessus dans un objet de niveau supérieur. Ceci n'est pas sans rappeler le mécanisme de réification dont parle Sfard (1991) lorsqu'elle met en avant la dualité processus-objet des concepts mathématiques. Sfard prend également l'exemple des nombres : ainsi les nombres relatifs $\mathbf{Z}$ sont-ils apparus tout d'abord en tant que processus ( $-b$ coincide avec l'opération de soustraction d'un entier naturel $b$ ) avant d'être 
reconnu comme des objets à part entière, dans ce qu'elle appelle la " phase structurale », par rapport à la «phase opératoire » (operational).

Notre contexte structuraliste se situe par contre à un niveau supérieur d'organisation, par rapport au contexte de Sfard : ce ne sont pas les opérations sur les entiers relatifs qui nous concernent, mais la structure de $(\mathbf{Z},+)$ en tant que groupe. La formulation de Cavaillès-Benis-Sinaceur est très intéressante à cet égard : le passage de la « liaison-acte »à la « liaisontype » est le passage d'un processus à un type d'objet. Cela éclaire la différence avec le contexte de Sfard : le processus d'abstraction (double) dont nous avons parlé conduit non pas à un objet mais à un type d'objet (la structure de groupe). Cela clarifie aussi un point commun, qui pouvait nous échapper dans notre formulation objets / structures : le processus d'abstraction-idéalisation mobilise également la modalité processus de l'objet duel « addition des entiers relatifs $»$. Des études approfondies sont nécessaires afin de comprendre comment s'établit une « liaisontype » en relation avec la dualité processus / objet de la relation elle-même et le niveau de réflexivité qui est engagé (nécessité et rôle d'un métalangage, afin d'exprimer ces propriétés formelles). À la différence du contexte de Sfard, l'apport de la dimension prédicative (Vergnaud, 2002) de la connaissance n'est pas à sous-estimer : les propriétés formelles des relations doivent vérifier la contrainte que certaines assertions sur les objets en soient des conséquences logiques. L'établissement des liaisons-types, qui placent l'objet au sein d'un paradigme, s'effectue également en relation avec d'autres objets qui contribuent à susciter le paradigme. Tout ceci dépasse la simple encapsulation d'un processus.

\section{«Phénoménologie didactique » Des STRUCTURES MATHÉMATIQUES}

\section{L'apport de Freudenthal et le cas de l'algèbre classique}

L'expression "phénoménologie didactique » (didactical phenomenolgy) est de la plume de Freudenthal (1983) :

"Our mathematical concepts, structures, ideas have been invented as tools to organise the phenomena of the physical, social and mental world. Phenomenology of a mathematical concept, structure, or idea means describing it in its relation to the phenomena for which it was created, and to which it has been extended in the learning process of mankind [...], it is didactical phenomenology, a way to show the teacher the places where the learner might step into the learning process of mankind." (p.ix.)

Nous allons, dans ce qui suit, détailler la pensée de Freudenthal afin d'expliquer la citation précédente. Nous rendrons compte également de l'interprétation qu'en ont faite certains didacticiens contemporains lors de travaux récents menés en didactique de l'algèbre élémentaire, au niveau de l'enseignement de second degré (Eugenio, Rojano \& Puig, 2008).

Pour Freudenthal, l'analyse phénoménologique d'un concept mathématique (ou d'une structure mathématique, au sens large pour Freudenthal, ou au sens précis de structure algébrique pour ce qui nous concerne), consiste à repérer le phénomène dont ce concept ou cette structure est le moyen ou le principe d'organisation et à décrire les relations entre concept-structure et phénomène. Ce couplage entre phénomène et principe d'organisation peut se situer à différents niveaux : celui du monde réel (Freudenthal parle de l'espace physique, de la sphère sociale), le formalisme mathématique étant engagé dans une modélisation du réel, ou à un niveau supérieur où ces formalisations, qui ont servi de principe d'organisation au niveau inférieur, sont prises pour objet (de la pensée) et entrent en tant que phénomène dans un nouveau couple phénomène / principe d'organisation. L'activité mathématique génère ainsi son propre contenu. Ce processus est une forme de thématisation.

L'analyse phénoménologique des concepts est d'emblée située par Freudenthal comme une composante du travail didactique : elle fournit des points d'ancrage à l'apprentissage, connaissance précieuse pour l'enseignant lorsqu'il s'agit de concevoir des situations d'apprentissage. Il s'agit là d'une fonction bien connue de l'analyse épistémologique, en relation avec le travail d'ingénierie didactique (Artigue, 1990), à cette différence près que Freudenthal situe l'épistémologie dans le courant phénoménologique. Il convient cependant de distinguer la phénoménologie didactique de la phénoménologie philosophique (Patras, 2005) :

«Pour en revenir à la phénoménologie [philosophique, dans ses rapports aux mathématiques], 
elle repose, au moins pour le type de questions considérées ici, sur l'idée que les mécanismes de création éidétiques (en termes plus classiques, grossièrement équivalents, que les actes de synthèse) sont organisés et relèvent d'une logique spécifique. Des notions comme celles d'horizon ou de variation éidétique permettent de décrire cette organisation. Un des moments cruciaux de cette description est l'édification préalable d'un modèle à un niveau ontologiquement pur, c'est-à-dire indépendamment de toute détermination psychologique ou empirique, de la distinction opérée classiquement entre la conscience et son objet. » (p. 409.)

Alors que la phénoménologie philosophique va rechercher des processus purs de la pensée à l'œuvre dans la sphère de la création mathématique, en relation avec la structure intentionnelle de la conscience, la phénoménologie didactique va questionner l'acquisition des concepts en tant que processus d'apprentissage dans un environnement didactique tenant compte, dans sa modélisation, des déterminations psychologiques et du milieu (Brousseau, 1998). Pour ces raisons, Freudenthal (1983) introduit une nouvelle distinction :

\footnotetext{
"In the present book I stress one feature more explicitly: mental objects versus concept attainment. Concepts are the backbone of our cognitive structures. But in everyday matters, concepts are not considered as a teaching subject. [...] Children learn what is number, what are circles and what is adding, what is plotting a graph. They grasp them as mental objects and carry them out as mental activities." (p.x.)
}

La chronologie naturelle de l'apprentissage passe ainsi, pour Freudenthal, par l'enseignement d'objets mentaux avant celui des concepts correspondants. Eugenio et al. (2008, chap. 2) tentent de préciser la nature et le fonctionnement des objets mentaux, en lien avec les concepts : par opposition à ces derniers, les objets mentaux se situent du côté des conceptions car ils sont liés au sujet épistémique. Eugenio et al. (2008) les mettent en correspondance avec le « champ sémantique individuel " (personal semantic field), défini comme la partie de la totalité des usages du concept, quel qu'en soit le contexte, qu'un apprenant a rencontrés et dont il a fait sens. Les objets mentaux appartiennent donc au concept image $e^{5}$ par rapport au concept definition, selon la distinction introduite par Tall et Vinner (1981). Ils apparaissent, tout comme les concepts, comme des moyens d'organiser les phénomènes et sont constitués au sein de chaînes de phénomènes-moyens d'organisation. Comme ils précèdent les concepts dans leur genèse, ils ne correspondent pas au concept definition image de Tall et Vinner (la représentation personnelle que se fait l'apprenant du concept definition) mais davantage à son complémentaire au sein du concept image, même si les objets mentaux peuvent être amenés à évoluer lors de la définition des concepts. En ce sens, ils précisent, par rapport aux travaux de Tall et Vinner, la relation entre concept definition et concept image, non pas dans le mouvement des concepts aux représentations, mais dans le mouvement inverse.

La constitution d'un objet mental se rapporte à la dimension opératoire de la connaissance alors que la constitution du concept se situe davantage du côté prédicatif (selon les distinctions soulignées par Vergnaud, 2002). À cet égard, Eugenio et al. (2008) écrivent :

"Constituting a mental object implies being able to give an account with it of all the uses in all contexts or being able to organize all the corresponding phenomena, in which case the mental object is well constituted. [...] Acquiring the concept implies examining how it was established in mathematics organized locally or globally in a deductive system." (p. 50.)

On notera cependant que Eugenio et al. (2008) requièrent, par rapport à Vergnaud (2002), un certain niveau de réflexivité, alors que la connaissance opératoire de Vergnaud s'exerce " en acte». Eugenio et al. (2008) mettent également en relation épistémologie génétique et épistémologie historique : un concept mathématique, en tant que processus historique, est le résultat d'un processus de définition, lequel incorpore le concept dans un système déductif qui en restreint le champ sémantique associé. Ainsi les concepts apparaissent-ils comme reliés à une partie des objets mentaux, celle qui est engagée d'un point de vue sémantique par le processus de définition du concept (Eugenio et al., 2008, p. 49). Cette réflexion souligne également l'importance, pour la conceptualisation, qui est à accorder au processus de définition lui-même, dans ses rapports aux intuitions et représentations qui voient naître les concepts (les objets mentaux) et ses rapports à la preuve (dialectique de Lakatos). D'un point de vue didactique, cela nécessite de considérer la définition en tant qu'activité, dans l'esprit des travaux d'Ouvrier-Buffet (2013). 
Notons également que les concepts ne sont pas amenés à remplacer les objets mentaux, lorsque le processus de définition est achevé. Les processus de développement d'objets mentaux et d'acquisition de concepts sont enchevêtrés. À la suite de Freudenthal, Eugenio et al. (2008, p. 50) donnent l'exemple de la notion de courbe en topologie : si la notion intuitive de courbe est bien pauvre par rapport à la définition de Jordan, le concept de Jordan mobilise d'autres objets mentaux liés à la notion d'espace et de variétés de grande dimension.

Dans la démarche de Eugenio et al. (2008), l'analyse phénoménologique des concepts a pour objectif l'élaboration de modèles d'enseignement de l'algèbre élémentaire. L'approche phénoménologique apparâit naturelle, du fait du rôle du symbolisme algébrique en tant que système de signes modélisant des grandeurs dans des situations d'action sur le réel. L'algèbre est donc présentée comme un moyen d'organisation des phénomènes et se déploie dans un jeu dialectique entre syntaxe (manipulation du système de signes selon des règles en cours d'élaboration) et sémantique (interprétation de ces systèmes de signes en relation avec les objets et démarches d'action, ce qui permet de donner du sens et d'exercer un certain contrôle sur les manipulations syntaxiques) :

\footnotetext{
"One gateway to the learning of algebra is modeling. In this kind of approach the emphasis is on the role of the sign system of algebra as a means to express relations between variables that correspond to phenomena or situations in the physical world, and the corresponding didactic paths contemplate the complete cycle: (1) translation of "concrete" situations or situations expressed in natural language (word problems) to algebraic code; (2) analysis of relations between variables, based on manipulation of the algebraic expressions produced (syntactic level); and (3) interpretation of the "concrete" situation in the light of the results of the work with algebraic syntax. The argument in favor of the virtues of this approach to algebra is that in step (1) meaning is given to algebraic expressions, and in steps (2) and (3) the syntactic manipulation of those expressions becomes meaningful." (p. 38.)
}

\section{Le cas de l'algèbre abstraite}

Il s'agit maintenant d'adapter ce cadre au contexte de l'enseignement supérieur, en l'occurrence l'enseignement des structures algébriques, en tenant compte des spécificités de ce savoir par rapport à l'algèbre classique.

Une phénoménologie didactique du structuralisme mathématique, comme nous l'avons vu, doit considérer deux niveaux : tout d'abord, le niveau de la structure (de groupe, d'anneau, etc.), qui apparaît en tant que principe organisateur de phénomènes impliquant des objets de niveau inférieur, voire du monde réel (ou à peine idéalisés) ; ensuite, le niveau du métaconcept de structure lui-même, qui est appelé à jouer un rôle architectural dans l'élaboration des théories mathématiques, en relation avec la méthodologie structuraliste.

Le premier couple organisateur, phénomène-principe, régit donc, par rapport au schéma en niveaux de la figure 1 , la relation entre les niveaux 1 et 2 , tandis que le second couple met en jeu les niveaux 2 et 3 . La présence de ce double niveau constitue un challenge important pour les étudiants, que nous avons appelé le "challenge de la pensée structuraliste » (Hausberger, 2012).

Les structures algébriques sont introduites dans l'enseignement universitaire par leur définition axiomatique, donc par les concepts, plutôt que par le biais des objets mentaux qui seraient susceptibles de leur correspondre. En référence à la chronologie préconisée par Freudenthal, il s'agit d'une « inversion didactique », que ce dernier dénonce. À moins que le discours de Freudenthal ne soit applicable qu'aux mathématiques du secondaire, les spécificités de la pensée mathématique avancée qui implique des concepts FUGS (Robert, 1987) faisant que ces derniers nécessiteraient une autre approche. Dans Hausberger (2016b), nous proposons une approche «bottom-up» de l'enseignement de la théorie des anneaux, par opposition à l'approche "top-down » majoritaire (c'est l'introduction frontale des structures dont nous avons parlé), qui se donne le temps de construire les concepts en mettant en avant la dimension outil (Douady, 1986) et en développant des stratégies dédiées à enrichir le milieu. Pour suivre la trace de Freudenthal, il s'agit d'étudier la possibilité de s'appuyer sur des objets mentaux dans l'apprentissage de l'algèbre abstraite afin de retrouver une certaine " phénoménalité » des structures algébriques. Nous évoquerons dans la conclusion nos travaux d'ingénierie didactique qui visent à implémenter cette stratégie.

Le processus de définition, dans le cas de l'algèbre abstraite, relève à la fois d'un processus d'abs- 
traction-idéalisation (aboutissant à l'énonciation des propriétés formelles des relations) et d'un travail d'analyse-synthèse (le mathématicien structuraliste parcourt les preuves portant sur les objets de niveau inférieur afin d'en isoler les "ressorts», qui sont posés en principes, puis vérifie que la théorie en est une conséquence logique). Pour autant, la genèse historique permet d'apercevoir le rôle important, bien qu'il soit difficile d'en rendre compte, joué par les intuitions portées par les objets et les représentations mentales que s'en construisent les mathématiciens. Le lien avec des représentations mentales explicites est donc plus ténu que dans le cas de l'algèbre classique. Comment imaginer une « activité de définition » dans ces conditions? Notre proposition est d'utiliser l'axiomatique dans son rôle de modélisation du réel, mais en ne s'arrêtant pas dans la formalisation au premier niveau d'abstraction qui est celui de l'algèbre classique (on met en avant des opérations sur des objets représentés symboliquement) : il s'agit de décrire les propriétés formelles de ces opérations, autrement dit d'établir une mini-théorie au sens de la méthodologie des sciences déductives de Tarski (1930/1972).

Enfin, dans notre étude de l'algèbre abstraite, nous avons décidé d'opérer un autre choix que celui de Eugenio et al. (2008), qui définissent les objets mentaux comme une partie des contextes d'usage des concepts. Nous suivons Duval (1995), qui distingue différents types de représentations: les représentations mentales - parmi lesquelles nous situons les objets mentaux de Freudenthal ${ }^{6}$ - sont des représentations conscientes internes qui remplissent une fonction d'objectivation. Mais elles sont, par essence, inaccessibles à l'investigation du didacticien et c'est pourquoi nous nous centrerons sur les représentations externes, les représentations sémiotiques, qui remplissent également une fonction d'objectivation, ainsi que d'expression et de traitement (Duval, 1995). Nous appellerons donc désormais objet mental externalisé toute représentation sémiotique introduite par un sujet épistémique dans un processus d'apprentissage d'un concept mathématique, représentation qui remplit une fonction d'objectivation en association avec une représentation mentale dont elle vise l'expression, et qui est amenée, notamment par une succession de traitements, à évoluer en une représentation sémiotique reconnue comme désignant le concept.

Il existe une limitation du fait du décalage entre les représentations mentales d'un sujet et les repré- sentations sémiotiques qu'il est capable de produire. Par ailleurs, la notion d'objet mental externalisé repose également sur le postulat, de nature psychologique, d'existence d'objets mentaux associés par le sujet à un concept mathématique tel qu'une structure algébrique. La reconnaissance de telles représentations sémiotiques est donc autant problématique que la reconnaissance des objets mentaux eux-mêmes. Cependant notre définition éclaire le processus qui conduit de l'objet mental au concept, que nous nous proposons d'analyser du point de vue de la production de signes par l'apprenant.

Duval (1995) nous éclaire également sur une différence importante à saisir entre représentation mentale et représentation sémiotique : ces dernières possèdent un « degré de liberté nécessaire à tout traitement d'information », que les premières ne possèdent pas, car elles «peuvent être appréhendées soit uniquement sous l'aspect du représentant, soit uniquement sous celui de ce qu'il représente ", à la différence des premières qui « se limitent à une seule visée, celle de ce qui est représenté » (Duval, 1995, p. 29). Pour autant, les représentations mentales peuvent également être amenées à se développer, notamment grâce à l'acquisition et à l'intériorisation de représentations sémiotiques. Un objet mental associé à une structure algébrique est donc, en première instance, directement lié à la sémantique de la structure, c'est-à-dire à l'un des objets de niveau inférieur dont la structure est issue par le processus d'abstraction-idéalisation. Il s'agira d'en détecter la trace en terme d'objet mental externalisé, puis d'observer comment sa nature de représentation sémiotique lui permet d'évoluer vers une représentation efficace capable de se prêter à ces traitements qui font la force du langage algébrique et qui se traduit, dans le cas de l'algèbre abstraite, par la possibilité d'une combinatoire au sein de la théorie de structure concernée.

Duval (1995) met également en avant une autre force des représentations sémiotiques :

« En raison de la diversité des systèmes sémiotiques, les représentations sémiotiques permettent d'avoir une variété de représentations pour un même objet, cette variété se révélant décisive à la fois du point de vue de la fonction de traitement et du point de vue de la conceptualisation. » (p. 30.)

Cet aspect entre d'autant plus en résonance avec les concepts de l'algèbre abstraite que ces derniers 
sont des concepts unificateurs : une structure algébrique introduit un point de vue unifié sur les objets de niveau inférieur. La conceptualisation d'une telle structure passe donc par la prise de conscience que ces différents objets sont des instances d'un même principe organisateur, ce qui nécessite une pluralité de représentations sémiotiques et la possibilité de constater qu'elles sont toutes « congruentes ». Dans ce qui suit, nous discuterons cet apport fondamental de la sémiotique. En définitive, après acquisition et intériorisation de ces propriétés de congruence, un objet mental associé à une structure algébrique apparaîtrait davantage comme un « cristal », un objet aux multiples facettes, permettant des « transferts d'intuitions ».

\section{LAPPORT DE LA SÉMIOTIQUE}

\section{Sémiotique des objets mathématiques}

La sémiotique est bâtie sur le principe que ce sont les systèmes de signes qui donnent accès aux objets mathématiques, de même qu'il est nécessaire de disposer d'un langage afin d'exprimer une idée. Nous utilisons dans cet article la sémiotique de Frege, qui introduit la distinction entre le sens et la dénotation d'un signe (Frege, 1892). La dénotation renvoie à l'objet que le signe désigne tandis que le sens est relié $\mathrm{au}$ « mode de donation» de l'objet. Par exemple, 5 et $2+3$ dénotent le même nombre, mais revêtent des sens différents, si bien que l'égalité $2+3=5$ renvoie au fait que 5 peut être vu comme le résultat d'un calcul, l'addition de 2 et de 3 .

Les signes mathématiques sont souvent polysémiques, mais le contexte est censé permettre de déterminer la référence de façon unique. Réciproquement, différents signes peuvent désigner le même objet, ayant ainsi des sens différents mais une dénotation commune. De cette façon, différentes représentations peuvent contribuer à éclairer différents aspects d'un objet. Des représentations sont reconnues comme désignant un unique objet lorsque l'on s'aperçoit que certaines transformations permettent de passer d'une représentation à l'autre, et réciproquement : ces transformations peuvent nécessiter des conversions d'un registre sémiotique à un autre ou bien se réduire à des traitements au sein d'un unique registre (Duval, 1995). En résumé, ainsi que le formule et l'illustre Winsløw (2004, p. 4), les objets mathéma- tiques se décrivent, du point de vue de la sémiotique, comme des « signes modulo des transformations préservant les objets (object preserving transformations ou OPT)» (Fig. 3).

\section{Figure 3.}

Schématisation sémiotique du processus de conceptualisation des objets mathématiques

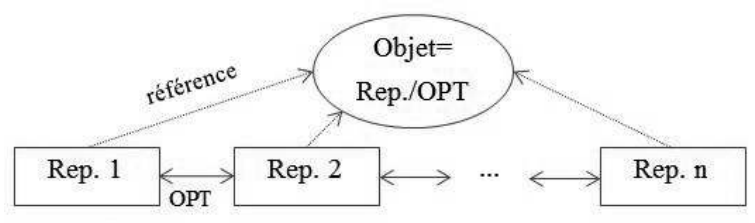

Winsløw (2004, p. 3) donne l'exemple, très éclairant, du cercle unité et de ses diverses représentations afin d'illustrer ce schéma ainsi que les différents traitements et conversions entre registres. Enfin, Duval montre dans ses travaux l'importance de la pluralité des représentations et de la diversité des registres dans le processus de conceptualisation. En référence à la dualité objet-processus des notions mathématiques soulignée par Sfard (1991), certaines de ces représentations mettrons en avant davantage la dimension structurelle (objet) et d'autres la dimension opératoire (processus).

\section{Syntaxe et sémantique}

Les signes mathématiques sont organisés en formules et en énoncés qui sont construits en se conformant à des règles syntaxiques strictes. D'un point de vue logique, une définition par axiomes est une "phrase ouverte », sans valeur de vérité, qui peut être satisfaite ou non lorsqu'on assigne aux différentes variables des valeurs prises au sein d'un certain univers de discours : il s'agit là du point de vue sémantique sur la vérité introduit par Tarski (1944) et dont Durand-Guerrier (2003) montre l'apport pour l'analyse didactique du raisonnement mathématique et des preuves.

À la suite de Tarski, nous appelons modèle de la structure (au sein d'un certain univers de discours ou théorie hôte) toute donnée qui satisfait la définition axiomatique de la structure. Les modèles constituent le contenu sémantique de la structure mathématique axiomatique, son extension en tant que concept (au 
sens de Frege, voir paragraphe suivant), par rapport au système d'axiomes qui la définit syntaxiquement.

Par exemple, $\mathbf{Z} / 4 Z$, le groupe multiplicatif des racines quatrième de l'unité et le groupe des isométries du rectangle sont trois modèles de l'axiomatique de groupes. Ils correspondent tous les trois à la donnée d'un ensemble (en l'occurrence de cardinal 4) muni d'une loi vérifiant les axiomes de la structure de groupe. Plus précisément, les deux premiers sont des modèles de groupe cyclique d'ordre 4 (on rajoute l'axiome d'existence d'un générateur d'ordre 4), alors que le troisième ne l'est pas.

\section{Figure 4.}

Processus d'enrichissement sémantique d'une structure définie syntaxiquement

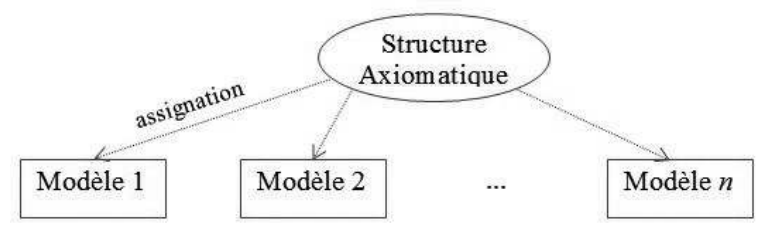

Points de vue syntaxique et sémantique sur le processus d'abstraction-idéalisation

Le verbe abstraire possède trois sens différents : tout d'abord, il signifie "faire abstraction de ensuite, « isoler (d'un contexte) »; enfin, « construire (un concept) ». Bien qu'il s'agisse de trois actions différentes, ces sens sont susceptibles d'être combinés comme c'est le cas dans l'algèbre abstraite : les mathématiciens font abstraction de la nature particulière des objets et isolent les propriétés formelles des relations afin de construire la structure comme concept abstrait.

Cette description du processus d'abstractionidéalisation correspond à ce que nous appelons la « version syntaxique » de ce processus car il aboutit à la production d'un système syntaxique exprimant la logique des relations. Ce processus procède d'un mouvement allant du sémantique au syntaxique. La description des propriétés formelles des relations sous forme de système axiomatique s'apparente également, d'une certaine manière, à un phénomène d'encapsulation d'un processus (Sfard, 1991), celui de l'énumération des possibilités opératoires de la relation, jusqu'à en épuiser la possibilité (conden- sation) et produire un motif formel (réification). La notion de relation convoque souvent celle de générateur, qui renvoie également à une dimension opératoire, algorithmique de processus itéré, qui est appelé à être encapsulé dans un objet.

La «version sémantique » du principe d'abstraction-idéalisation en jeu dans les structures algébriques, en tant que processus générateur de concept, est appelé par les philosophes-logiciens «principe d'abstraction ». Il a été utilisé, par exemple, par Frege (1884) pour définir les nombres cardinaux. Afin de présenter à son lecteur cette idée révolutionnaire, Frege donne l'exemple très éclairant de la direction d'une droite, laquelle est définie en tant que la classe de toutes les droites qui sont parallèles à la droite donnée. Ce principe a été formalisé plus tard par Russell (1903) : dire que des « choses sont égales » parce qu'elles partagent une propriété commune, réduisant ainsi à un seul élément toute une classe, nécessite que la relation qui traduit cette propriété soit symétrique, réflexive et transitive (en d'autres termes, soit une relation d'équivalence). Ce principe d'abstraction a été utilisé par Carnap afin de définir la notion de structure dans le contexte des systèmes ensemble-relation. Cette version du principe d'abstraction-idéalisation nous permet ainsi de définir une structure comme un ensemble de classes de modèles, ce qui s'apparente bien à une description sémantique. La notion-clé est celle de relation d'équivalence qui est porteuse d'une dualité syntaxe-sémantique : se donner une relation d'équivalence est équivalent à se donner une partition. La relation d'équivalence en jeu ici est la relation d'isomorphisme; cette seconde interprétation du principe d'abstraction-idéalisation constitue ainsi un mouvement du syntaxique au sémantique.

Finalement, le principe d'abstraction-idéalisation est porteur d'une dialectique syntaxe-sémantique. Celle-ci est liée à la dialectique objet-structures. La version syntaxique du processus d'abstraction-idéalisation engage un domaine phénoménal principal dont la structure est issue, en tant que principe organisateur abstrait, dans un mouvement des objets à la structure. Dans un second mouvement de la structure aux objets, la considération de la pluralité des modèles, en instaurant un jeu dialectique entre les deux niveaux objets-structure, invite, à travers la version sémantique du principe d'abstraction-idéalisation, à convoquer en fait plusieurs domaines de phénomènes. Dans cette dialectique concret-abstrait, 
les classes d'équivalence constituent un intermédiaire entre le domaine sémantique concret des objets et celui syntaxique abstrait de la structure. Le prix à payer est la transition des éléments aux classes. La conceptualisation de ces classes engage un processus de réification (au sens de Sfard, 1991), ainsi que nous allons le constater.

\section{Les « objets structuraux »}

Dans une dispute célèbre avec Hilbert (Blanchette, 1996), Frege argumente contre la légitimité des définitions abstraites par système axiomatique. Une de ses objections concerne la polysémie intrinsèque de ces définitions : en termes sémiotiques, une définition axiomatique en tant que signe possède de multiples références, les différents modèles de la structure axiomatique. Dans le cas de ces définitions abstraites, le contexte ne donne aucune information sur la dénotation, justement parce qu'il est abstrait (au premier sens de ce verbe)!

Afin de construire un concept de structure (de groupe, d'anneau, etc.) dans un tel contexte de références multiples, il s'avère nécessaire de recourir aux « transformations qui conservent la structure » (structure preserving transformations ou SPT), appelées isomorphismes, qui sont définies en tant que bijections préservant les relations. Ceci nous permet d'associer à une structure axiomatique ses « objets structuraux » (notre terminologie), c'est-à-dire les classes d'isomorphismes de modèles ou encore les modèles modulo SPT, de la même façon que les objets mathématiques sont construits à partir de représentations modulo OPT, en utilisant le principe d'abstraction (Fig. 5).

Figure 5.

Processus de conceptualisation des objets structuraux fondé sur la version sémantique du processus d'abstraction-idéalisation

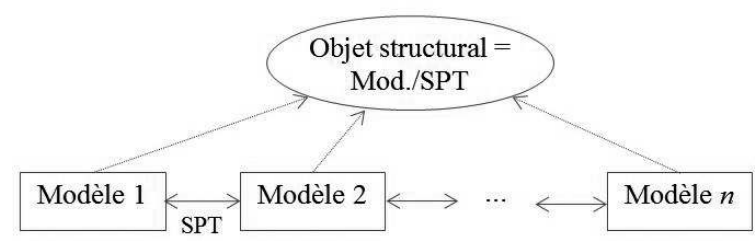

Par comparaison avec le schéma de Winsløw, il est important de souligner que les flèches en pointillé ne représentent pas la dénotation d'un signe, mais uniquement des " applications de passage au quotient $»$. Afin de se conformer au point de vue sémiotique, il s'agit de remplacer chaque modèle par une de ses représentations et les SPT par des transformations préservant l'objet structural (structural object preserving transformations ou SOPT). Les flèches en pointillé représentent alors bien une dénotation lorsque le contexte indique une perspective structurale : par exemple, $\mathbf{Z} / 2 \mathbf{Z} \times \mathbf{Z} / 2 \mathrm{Z}$ et « le groupe des isométries du rectangle » sont susceptibles de désigner tous deux le groupe de Klein V4, en tant que groupe abstrait. On peut également écrire $V 4=\left\langle a, b \mid a^{2}=b^{2}=(a b)^{2}=1\right\rangle$ (présentation par générateurs et relations) afin d'en donner une description plus abstraite et syntaxique. Néanmoins, parce que les mathématiciens prennent soin en général de bien distinguer une classe et l'un de ses représentants, beaucoup d'auteurs préfèrent utiliser le signe V4 pour désigner le groupe et disent que ce dernier est isomorphe au groupe des isométries du rectangle ou au quotient du groupe libre à deux générateurs par les relations $a^{2}=b^{2}=(a b)^{2}=1$. L'idée derrière les objets structuraux est qu'une forme de réification doit avoir lieu afin de construire un concept (Sfard, 1991, p. 19): "Reification is defined as an ontological shift - a sudden ability to see something familiar in a totally new light. " Afin que ce phénomène se produise, nous faisons l'hypothèse qu'une pluralité de modèles est nécessaire, empruntés à différents domaines des mathématiques et représentés dans une variété de registres sémiotiques. De façon analogue au contexte de Winsløw, la coordination de ces représentations à travers les SOPT devrait être cruciale afin de générer chez l'apprenant un schéma conceptuel de l'object structural. Ce travail sur les représentations doit ouvrir la possibilité d'extraire des modèles (templates) un motif (pattern) (Resnik, 1997). Néanmoins, à la différence du contexte de Winsløw, une représentation d'un modèle, en tant que signe, est susceptible de désigner à la fois le modèle et l'objet structural (dans un contexte où les deux se présentent), alors qu'il est souhaitable de maintenir une distinction mathématique entre les deux. Résoudre ce problème requiert une médiation plus directe de l'objet structural, par le biais d'un nouveau signe (que l'on souhaiterait davantage opératoire qu'un simple surlignage indiquant une classe). 
La pluralité des représentations sémiotiques permet également de mobiliser des processus de traitements internes différents selon les registres: dans le cadre du registre graphique, la reconnaissance d'un isomorphisme peut s'apparenter à un processus visuel de reconnaissance d'une congruence de formes, en relation avec l'étymologie même du terme isomorphisme, comparativement à d'autres registres dans lesquels le lien d'isomorphie, moins aisé à établir, requiert des manipulations syntaxiques en relation avec la définition formelle d'isomorphisme. Dans cet ordre d'idées, nous avons déjà souligné le potentiel de la représentation des relations en termes de graphes.

\section{CONCLUSION}

Notre étude épistémologique de l'algèbre abstraite, en appui sur le travail des historiens et des philosophes, nous conduit à placer au cœur de la pensée mathématique à l'œuvre dans ce champ la dialectique entre objets et structures. Cette structure d'organisation des objets résulte d'une dialectique concret-abstrait mettant en jeu un mouvement double d'abstraction-idéalisation et d'abstractionthématisation qui opèrent transversalement.

L'abstraction-idéalisation conduit d'une "liaison-acte » à une « liaison-type », d'un processus à une structure. Nous avons éclairé ce phénomène à l'aide de distinctions opérées par Carnap et discuté ses liens, d'un point de vue cognitif, avec la dualité processus / objet de Sfard (1991). L'abstractionthématisation est indissociable d'un point de vue métacognitif sur le premier procédé d'abstractionidéalisation et conduit à la méthodologie structuraliste. Nous soutenons que son étude nécessite un point de vue réflexif sur différentes structures, lequel favorise l'approfondissement de ces théories et l'apprentissage de nouvelles théories structuralistes.

Le point de vue "phénomènes-moyens d'organisation de ces phénomènes » sur les structures mathématiques, soutenu par Freudenthal, est l'une des entrées importantes dans l'algèbre classique, en rapport avec la fonction du symbolisme algébrique. Elle a donné lieu à des stratégies d'apprentissage qui ont fait leur preuve et qui tendent à instaurer un fonctionnement dialectique entre syntaxe et sémantique. Nous avons étendu cette approche au cas de l'algèbre abstraite, en soulignant ses spécificités et en dégageant et distinguant des aspects syntaxiques et sémantiques, ainsi que leurs articulations. Le principe d'abstraction contient, par essence, une dualité syntaxe-sémantique, à travers la notion de relation d'équivalence. Cela consacre l'importance de la notion d'isomorphisme en algèbre abstraite.

Freudenthal met en avant le développement d'objets mentaux avant l'acquisition des concepts. Les témoignages des mathématiciens soulignent également le rôle des intuitions alors que les théories structurales apparaissent essentiellement comme des architectures logiques. Bien que la théorie des groupes tisse des liens profonds avec la géométrie, donc avec le monde phénoménal, peu de travaux d'ingénierie didactique, parus dans des revues de recherche et portés à notre connaissance, articulent l'apprentissage de la structure de groupe avec la construction d'objets mentaux, dans l'esprit de Freudenthal. Un des rares exemples est l'approche prospective de Larsen (2009), qui se propose de guider les étudiants dans l'élaboration ( « réinvention ») des concepts de groupe et d'isomorphisme via l'exploration des symétries de figures géométriques et la discussion du sens, pour deux groupes donnés, d'être « essentiellement les mêmes ». Il s'agit en quelque sorte d'accompagner le processus d'idéalisation, ce qui requiert de nombreuses interventions de l'instructeur et pose plusieurs difficultés non anticipées dont cet auteur rend compte. Par ailleurs, le rôle des objets mentaux n'est pas élucidé. Une difficulté à cela nous semble provenir du fait que, si l'action d'un groupe sur un ensemble (un espace géométrique) permet de fournir des informations sur ce groupe, se représenter la structure du groupe fait souvent appel à d'autres représentations phénoménologiques : par exemple, on ne reconnait pas que le groupe des isométries du rectangle et le groupe $\mathrm{Z} / 2 \mathrm{Z} \times \mathrm{Z} / 2 \mathrm{Z}$ sont isomorphes sur la base de l'image mentale du rectangle; le mathématicien se construit d'autres représentations, dont certaines lui sont propres, d'un produit de deux groupes cycliques d'ordre deux.

Nous avons donc inventé une nouvelle structure, la structure de banquet (Hausberger, 2016a), qui affiche d'emblée dans sa terminologie une référence phénoménale : le nom «banquet » évoque un ensemble de personnes assis autour de tables, notre esprit s'imagine un banquet de mariage. L'effet serait tout autre si la théorie avait été dénommée « théorie des schmilblicks $»$ ! Par ailleurs, notre choix d'inventer une nouvelle structure est également motivé 
Thomas Hausberger

par l'ambition d'éclairer le second niveau, « phénomène / principe organisateur », en discutant le métaconcept de structure. Il est pour cela important que les étudiants aient rencontré au préalable la structure de groupe et l'aient étudié selon la méthodologie structuraliste (classification des groupes de petits ordres, démonstration de théorèmes de structures, etc.), dans l'action (par rapport à l'activité des banquets qui introduit un niveau de réflexivité). En effet, le second mouvement d'abstraction (la thématisation) nécessite, comme nous l'avons vu, la considération de plusieurs structures, produites par le premier mouvement d'abstraction, l'idéalisation.

Afin de développer la conceptualisation d'une notion abstraite de structure, nous proposons la tâche de "classification » des différents modèles. Nous suivons ainsi la pensée de Piaget (1971, p. 16), pour qui « the abstraction is drawn not from the object that is acted upon, but from the action itself. It seems to me that this is the basis of logical and mathematical abstraction ».

Il est particulièrement intéressant d'observer, dans une telle tâche, les différentes représentations sémiotiques produites ainsi que les procédures de traitement au sein d'un registre et de conversion entre registres (Hausberger, 2016a). Parmi ces dernières figurent les objets mentaux externalisés. Comme nous l'avons souligné, le processus d'abstraction-idéalisation, dans sa version sémantique, est susceptible de sémiosis (Duval, 1995) : nous nous attendons à trouver des représentations sémiotiques liées à la conceptualisation d'objets structuraux, issus de ces objets mentaux externalisés. Les traitements et conversions observés nous renseignent sur les processus cognitifs en jeu dans le processus d'abstraction-idéalisation. Dualement, nous proposons d'engager également l'apprenant dans une activité de définition qui s'apparente à la constitution, par l'apprenant, d'une mini-théorie au sens de la méthodologie des sciences déductives de Tarski (1930/1972). Cela éclaire le processus d'abstraction-idéalisation dans sa dimension syntaxique. Nous dirigeons le lecteur vers notre note de synthèse de l'habilitation à diriger des recherches (Hausberger, 2016c) pour des analyses complémentaires et un compte-rendu des résultats obtenus, lesquels complètent et enrichissent les résultats partiels déjà parus (Hausberger, 2016a).

\section{NOTES}

1. Ceux de l'époque, bien sûr. Les manuels actuels ont en général totalement naturalisé le style bourbakiste et se gardent de tout commentaire métamathématique.

2. Dans le langage et avec les notations de la théorie des ensembles, deux ensembles munis d'une relation $(E, R)$ et $\left(E^{\prime}, R^{\prime}\right)$ sont isomorphes, par définition, s'il existe une bijection $\varphi: E \rightarrow E$ ' telle que pour tout couple $(x, y)$ d'éléments de $E$ on ait $x R y$ si et seulement si $\varphi(x) R^{\prime} \varphi(y)$. Une fonction constitue un cas particulier de relation, aussi Carnap placet-il sur le même plan l'isomorphisme et les relations sur les ensembles. Il va sans dire que le langage de la théorie des ensembles permet de clarifier considérablement le propos de Carnap.

3. Voir « Points de vue syntaxique et sémantique sur le processus d'abstraction-idéalisation »

4. Le substantif mixte qui apparaît ci-après et qui est utilisé par Lautman pour désigner les êtres mathématiques participe d'un positionnement philosophique que Benis-Sinaceur (2010) qualifie de «platonisme phénoménologique $»$.

5. Cette notion désigne pour Vinner la « totalité de la structure cognitive » associée au concept-definition, lequel correspond à la définition formelle telle qu'elle apparaît dans le texte du savoir.

6. Freudenthal (1983, p. 226), à propos des travaux de Piaget et Inhelder, commente le mot de représentation en évoquant les «child's representations, Vorstellungen in Kant's sense, intuitions as some others say, or "mental objects" as I prefer to call them ». 


\section{RÉFÉRENCES}

Artigue, M. (1990). Ingénierie didactique. Recherches en Didactique des Mathématiques, 9(3), 281-308.

Benis-Sinaceur, H. (1987). Structure et concept dans l'épistémologie mathématique de Jean Cavaillès. Revue d'histoire des sciences, 40(1), 5-30.

Benis-Sinaceur, H. (2010a). Idées : le platonisme phénoménologique d'Albert Lautman. Philosophiques, 37(1), 27-54.

Benis-Sinaceur, H. (2010, mars). Emmy Noether et l'école algébrique allemande dans le premier tiers du xxe siècle: mathématiques, style de pensée et philosophie. Communication aux Journées Noether, Lyon, France.

Blanchette, P. A. (1996, juillet). Frege and Hilbert on Consistency. The Journal of Philosophy, 93(7), 317-336. Récupéré de [http://www3.nd.edu/ pblanche/fregehilbert.pdf].

Bourbaki, N. (1998). Larchitecture des mathématiques. Dans Le Lionnais, F. (dir.), Les grands courants de la pensée mathématique (p. 35-47). Paris : Hermann.

Brousseau, G. (1998). Théorie des situations didactiques. Grenoble : La pensée sauvage.

Carnap, R. (1928). Der logische Aufbau der Welt. Berlin : Weltkreis.

Cavaillès J. (1994). Euvres complètes de philosophie des sciences. Paris : Hermann.

Chevallard, Y. (1989). Le passage de l'arithmétique à l'algèbre dans l'enseignement des mathématiques au collège, deuxième partie. Perspectives curriculaires : la notion de modélisation. Petit x, 19, 45-75.

Chevallard, Y. (2001). Les TPE comme problème didactique. Actes du séminaire national de didactique des mathématiques 2001 (p. 177-188). Paris : IREM de Paris 7 et ARDM.

Corry, L. (1996). Modern Algebra and the Rise of mathematical Structures. Bâle : Birkhaüser.

Corry, L. (2007). History of Algebra. Récupéré de [http:// www.tau.ac.il/ corry/publications/articles/pdf/algebra\%20EB.pdf].

Corry, L. (2016). Algebra. Dans Encyclopadia Britannica. Récupéré de [https://global.britannica.com/topic/ algebra/Structural-algebra].

Douady, R. (1986). Jeux de cadres et dialectique outilobjet. Recherches en didactique des mathématiques, 7(2), 5-31.

Durand-Guerrier, V. (2003). Logic and mathematical reasoning from a didactical point of view: A model-theoretic approach. Dans M. A. Mariotti (dir.), Proceedings of the Third Congres of the European Society for Research in Mathematics Education (Thematic Working Group 4). Récupéré de [http://www.mathematik.uni-dortmund. de/ erme/CERME3/Groups/TG4/TG4_Guerrier_ cerme3.pdf].

Durand-Guerrier, V., Hausberger, T., \& Spitalas, C. (2015). Définitions et exemples: Prérequis pour l'apprentissage de l'algèbre moderne. Annales de Didactique et de Sciences Cognitives, 20, 101-148
Duval, R. (1995). Sémiosis et pensée humaine. Registres sémiotiques et apprentissages intellectuels. Berne: Peter Lang.

Eugenio, F. Y., Puig, L., \& Rojano, T. (2008). Educational Algebra: A Theoretical and Empirical Approach. New York : Springer.

Frege, G. (1884). Die Grundlagen der Arithmetik. Breslau : W. Koebner.

Frege, G. (1892). Über Sinn und Bedeutung. Zeitschrift für Philosophie und philosophische Kritik, 100(1), 25-50.

Freudenthal, H. (1983). Didactical phenomenology of mathematical structures. Dordrecht : Reidel.

Granger G. G. (1994). Formes, opérations, objets. Paris : Vrin.

Gueudet, G. (2008). Investigating the Secondary-tertiary transition. Educational Studies in Mathematics, 67(3), 237-254.

Hasse H. (1930) Die moderne algebraische Methode. Jahresbericht der Deutschen Mathematiker-Vereinigung, 39, 22-34.

Hausberger, T. (2012). Le challenge de la pensée structuraliste dans l'apprentissage de l'algèbre abstraite : une approche épistémologique. Dans J.-L. Dorier $\&$ S. Coutat (dir.), Enseignement des mathématiques et contrat social : Enjeux et défis pour le $21^{\mathrm{e}}$ siècle (p. 425-434). Genève : Université de Genève.

Hausberger, T. (2013). On the concept of (homo) morphism: A key notion in the learning of abstract algebra. Dans B. Ubuz, C. Haser, M. A. Mariotti (dir.), Proceedings of the Eighth Congress of the European Society for Research in Mathematics Education (p. 23462355). Ankara : Middle East Technical University.

Hausberger, T. (2016a). Abstract algebra, mathematical structuralism and semiotics. Dans K. Krainer \& N. Vondrova (dir.), Proceedings of the Ninth Congress of the European Society for Research in Mathematics Education (p. 2145-2151). Prague : Faculty of Education, Charles University.

Hausberger, T. (2016b). Comment développer des praxéologies structuralistes en Algèbre Abstraite? Recherches en Didactique des Mathématiques, 36(1), 97-142.

Hausberger, T. (2016c). Enseignement et apprentissage de l'algèbre abstraite à l'université et premiers éléments d'une didactique du structuralisme algébrique : études croisées en didactique et épistémologique des mathématiques (Note de synthèse pour l'habilitation à diriger des recherches). Disponible en ligne à [https://hal. archives-ouvertes.fr/tel-01408565].

Kouki, R. (2008). Enseignement et apprentissage des équations, inéquations et fonctions au secondaire : entre syntaxe et sémantique (Thèse de doctorat, Université Claude Bernard Lyon 1). Récupéré de [https://halshs. archives-ouvertes.fr/tel-00346287v2/document]

Lajoie, C., \& Mura, R. (2004). Difficultés liées à l'apprentissage des concepts de sous-groupe normal et de groupe quotient, Recherches en Didactique des Mathématiques, 24(1), 45-80. 
Larsen, S. (2009). Reinventing the concepts of groups and isomorphism: The case of Jessica and Sandra. The Journal of Mathematical Behavior, 28, 119-137.

Lautman, A. (2006). Les mathématiques, les Idées et le Réel physique. Paris : Vrin.

Leron, U., \& Dubinsky, E. (1995). An abstract algebra story. American Mathematical Monthly, 102(3), 227-242.

Mac Lane, S. (1996). Structure in Mathematics. Philosophia Mathematica, 4(2), 174-183.

Nardi, E. (2000). Mathematics Undergraduates' Responses to Semantic Abbreviations, Geometric Images and Multi-level Abstractions in Group Theory. Educational Studies in Mathematics, 43(2), 169-189.

Ouvrier-Buffet, C. (2013). Modélisation de l'activité de définition en mathématiques et de sa dialectique avec la preuve. Étude épistémologique et enjeux didactiques [Note de synthèse pour l'habilitation à diriger des recherches]. Paris : Université Paris Diderot-Paris 7.

Patras, F. (2005). Phénoménologie et théorie des catégories. Dans L. Boi (dir.), Geometries of Nature, Living Systems and Human Cognition (p. 401-419). Singapour : World Scientific.

Patras, F. (2008). Carnap, l'Aufbau, et l'idée mathématique de structure. Dans J. Bouveresse \& P. Wagner (dir.), Mathématiques et expérience. L'empirisme logique à l'épreuve (1918-1940) (p. 33-54). Paris : Odile Jacob - Collège de France.

Piaget, J., \& Beth, E. W. (1961). Épistémologie mathématique et psychologie. Essai sur les relations entre la logique formelle et la pensée réelle. Paris: PUF.

Piaget, J. (1971). Genetic Epistemology. New York : W. W. Norton.

Robert, A. (1987, novembre). De quelques spécificités de l'enseignement des mathématiques dans l'enseignement post-obligatoire. Cahiers de didactique des mathématiques, 47.
Resnik, M. (1997). Mathematics as a Science of Patterns. Oxford : Oxford University Press.

Russell, B. (1903). The Principles of Mathematics. Cambridge : Cambridge University Press.

Sfard, A. (1991). On the dual nature of mathematical conceptions : Reflections on processes and objects as different sides of the same coin. Educational Studies in Mathematics, 22(1), 1-36.

Tall, D. O., \& Vinner, S. (1981). Concept Image and Concept Definition in Mathematics, with special reference to Limits and Continuity. Educational Studies in Mathematics, 12(2), 151-169.

Tarski, A. (1944). The semantic conception of truth and the foundation of semantics. Philosophy and Phenomenological Research, 4(3), 341-376.

Tarski, A. (1972). Concepts fondamentaux de la méthodologie des sciences déductives. Dans G. G. Granger (dir.), Logique, sémantique, métamathématique 19231944 (vol. 1, p. 67-116). Paris : Armand Colin.

Van der Waerden, B. L. (1930-1931). Moderne Algebra (2 vols). Berlin : Springer.

Vergnaud, G. (1990) La théorie des champs conceptuels. Recherches en didactique des mathématiques, 10(2), 133-170.

Vergnaud, G. (2002). Forme opératoire et forme prédicative de la connaissance. Dans J. Portugais (dir.), La notion de compétence en enseignement des mathématiques, analyse didactique des effets de son introduction sur les pratiques et sur la formation (p. 6-27). Montréal : Université de Montréal.

Weber, H. (1898). Traité d'algèbre supérieure. Paris : Gauthier-Villars.

Winsløw, C. (2004). Semiotics as an analytic tool for the didactics of mathematics. Nordic studies in Mathematics Education, 9(2), 81-100. 